\title{
Analyzing the Annual Maximum Magnitude of Earthquakes in Japan by Extreme Value Theory
}

\author{
Fumio Maruyama \\ Department of Sports and Health Science, Matsumoto University, Matsumoto, Japan \\ Email: fmaruya@nagoya-u.jp
}

How to cite this paper: Maruyama, F. (2020) Analyzing the Annual Maximum Magnitude of Earthquakes in Japan by Extreme Value Theory. Open Journal of Applied Sciences, 10, 817-824.

https://doi.org/10.4236/ojapps.2020.1012057

Received: November 2, 2020

Accepted: December 20, 2020

Published: December 23, 2020

Copyright (c) 2020 by author(s) and Scientific Research Publishing Inc. This work is licensed under the Creative Commons Attribution International License (CC BY 4.0).

http://creativecommons.org/licenses/by/4.0/

\begin{abstract}
One of the most important and interesting issues associated with the earthquakes is the long-term trend of the extreme events. Extreme value theory provides methods for analysis of the most extreme parts of data. We estimated the annual maximum magnitude of earthquakes in Japan by extreme value theory using earthquake data between 1900 and 2019. Generalized extreme value (GEV) distribution was applied to fit the extreme indices. The distribution was used to estimate the probability of extreme values in specified time periods. The various diagnostic plots for assessing the accuracy of the GEV model fitted to the magnitude of maximum earthquakes data in Japan gave the validity of the GEV model. The extreme value index, $\xi$ was evaluated as -0.163 , with a $95 \%$ confidence interval of $[-0.260,-0.0174]$ by the use of profile likelihood. Hence, the annual maximum magnitude of earthquakes has a finite upper limit. We obtained the maximum return level for the return periods of 10,20,50, 100 and 500 years along with their respective 95\% confidence interval. Further, to get a more accurate confidence interval, we estimated the profile log-likelihood. The return level estimate was obtained as $7.83,8.60$ and 8.99 , with a $95 \%$ confidence interval of $[7.67,8.06]$, $[8.32,9.21]$ and $[8.61,10.0]$ for the $10-, 100$ - and 500-year return periods, respectively. Hence, the 2011 off the Pacific coast of Tohoku Earthquake, which was the largest in the observation history of Japan, had a magnitude of 9.0, and it was a phenomenon that occurs once every 500 year.
\end{abstract}

\section{Keywords}

Extreme Value Theory, Generalized Extreme Value Distribution, Earthquakes

\section{Introduction}

Extreme value theory has emerged as one of the most important statistical disciplines for the applied science. Using the extreme value theory, the theoretical 
distribution and its population parameter that the maximum value follows are estimated from long-term observation data. And the maximum value or a large value that occurs once every 100 years can be predicted based on the estimated result. Extreme value techniques are also becoming widely used for portfolio adjustment in the insurance industry, risk assessment on financial markets, and traffic prediction in telecommunications [1].

Statistical approaches focused on extreme values have shown promising results in forecasting unusual events in earth sciences, genetics and finance. For instance, Extreme Value Theory (EVT) was developed in the 1920s [1] and has been used to predict the occurrence of events as varied as droughts and flooding [2] or financial crashes [3]. Application of extreme value modeling has been published in the fields of ocean wave modeling [4]; wind engineering [5]; biomedical data processing [6]; thermodynamics of earthquakes [7]; food science [8]; and public health [9].

Applications of extreme value statistics in geology can be found in the magnitudes of and losses from earthquakes [10] [11] [12]. The aim of this paper is to predict extreme earthquake events in Japan using extreme value theory.

\section{Data and Method of Analysis}

\subsection{Data}

We used the annual maximum magnitude of earthquakes in Japan for 1900-2019 by the Japan Meteorological Agency.

\subsection{Extreme Value Theory (EVT)—The Method of Block Maxima}

\subsubsection{General Extreme Value (GEV) Distribution}

When data are taken to be the maxima (or minima) over certain blocks of time (such as annual maximum precipitation), then it is appropriate to use the Generalized Extreme Value (GEV) distribution:

$$
G(z)=\left\{\begin{array}{l}
\exp \left\{-\left[1+\xi\left(\frac{z-\mu}{\sigma}\right)\right]^{-1 / \xi}\right\}, \text { for } \xi \neq 0 \\
\exp \left\{-\exp \left[-\left(\frac{z-\mu}{\sigma}\right)\right]\right\}, \text { for } \xi=0
\end{array}\right.
$$

where $\mu$ is a location parameter; $\sigma$ a scale parameter; and $\xi$ a shape parameter. $G$ is defined for all $\mathrm{z}$ such that $(1+\xi(z-\mu) / \sigma)>0$ for $\xi \neq 0$ and all $z$ for $\xi=0$. Three families of GEV distributions are defined depending on the value of $\xi$. For $\xi>0$ we get the Fréchet distribution with heavy tail, $\xi=0$, the Gumbel distribution with lighter tail and $\xi<0$ the Weibull distribution with finite tail.

A method for modelling the extremes of a stationary time series is the method of block maxima, in which consecutive observations are grouped into non-overlapping blocks of length $\mathrm{n}$, generating a series of $\mathrm{m}$ block maxima, $\mathrm{Mn}$, $1, \ldots, \mathrm{Mn}, \mathrm{m}$, say, to which the GEV distribution can be fitted for some large value of $n$. The usual approach is to consider blocks of a given time length, thus 
yielding maxima at regular intervals [1].

\subsubsection{Return Levels}

Once a GEV distribution is fitted to empirical observations, it becomes possible to estimate the probability of an event that has not been observed yet. Estimates of extreme quantiles of the annual maximum distribution are obtained by inverting Equation (1):

$$
z_{p}=\left\{\begin{array}{l}
\mu-\frac{\sigma}{\xi}\left[1-\{-\log (1-p)\}^{-\xi}\right], \text { for } \xi \neq 0 \\
\mu-\sigma \log \{-\log (1-p)\}, \text { for } \xi=0
\end{array},\right.
$$

where $G\left(z_{p}\right)=1-p$. The return level $z_{p}$ is associated with the return period $1 / p$, since to a reasonable degree of accuracy, the level $z_{p}$ is expected to be exceeded on average once every $1 / p$ years. More precisely, $z_{p}$ is exceeded by the annual maximum in any particular year with probability $p$ [1].

Modeling was performed using the ismev package in R for GEV calculations.

\section{Results and Discussion}

The annual maximum magnitude of earthquakes in Japan was shown in Figure 1 and was distributed approximately 6 to 8 . Figure 2 shows wavelet power spectrum of the annual maximum magnitude of earthquakes in Japan. For 1950-1970 the strong period of 5 years and for 1970-2010 the strong period of 10 years were observed.

The various diagnostic plots for assessing the accuracy of the GEV model fitted to the magnitude of maximum earthquakes data in Japan are shown in Figure 3. Neither the probability plot nor the quantile plot give cause to doubt the validity of the fitted model: each set of plotted points is near-linear. In the return level curve, the estimated curve is not close to linear, since the $\xi$ is not close to zero. Finally, the corresponding density estimate seems consistent with the histogram of the data. The various diagnostic plots give little reason to doubt the validity of the GEV model, and also show how the model extrapolates.

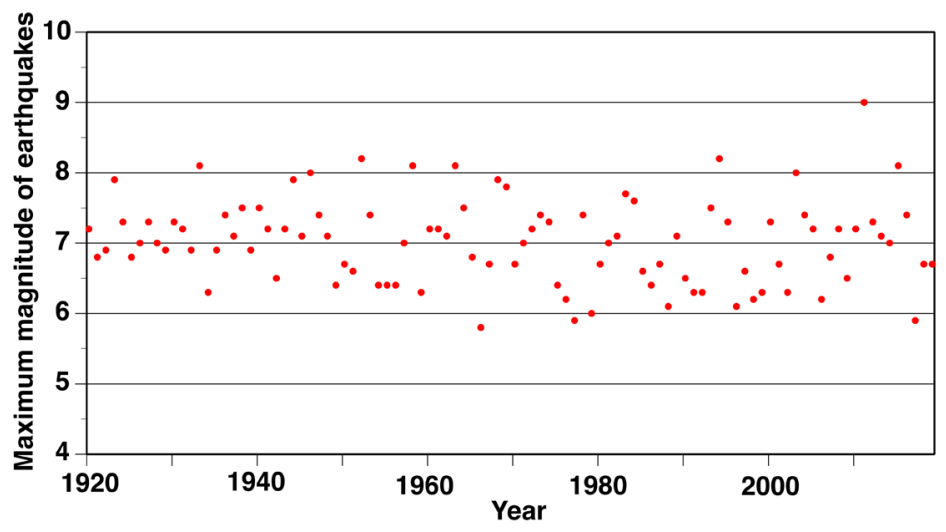

Figure 1. Plot of the annual maximum magnitude of earthquakes in Japan. 
Wavelet Power Spectrum

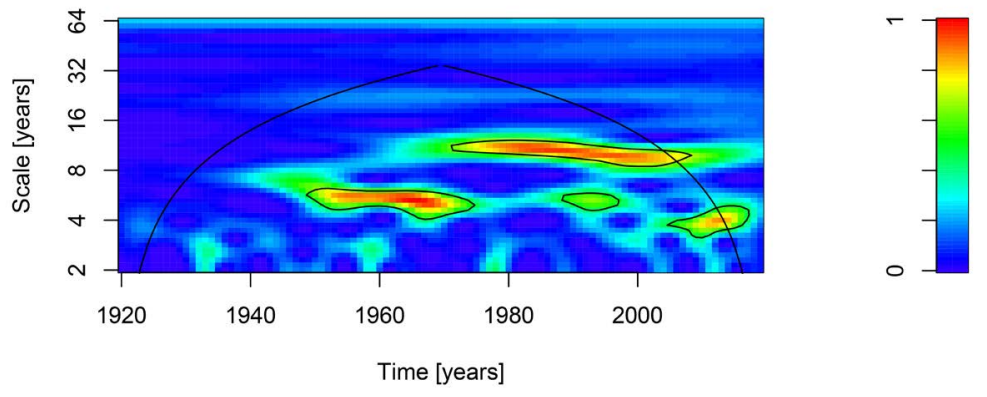

Figure 2. Wavelet power spectrum of the annual maximum magnitude of earthquakes in Japan.
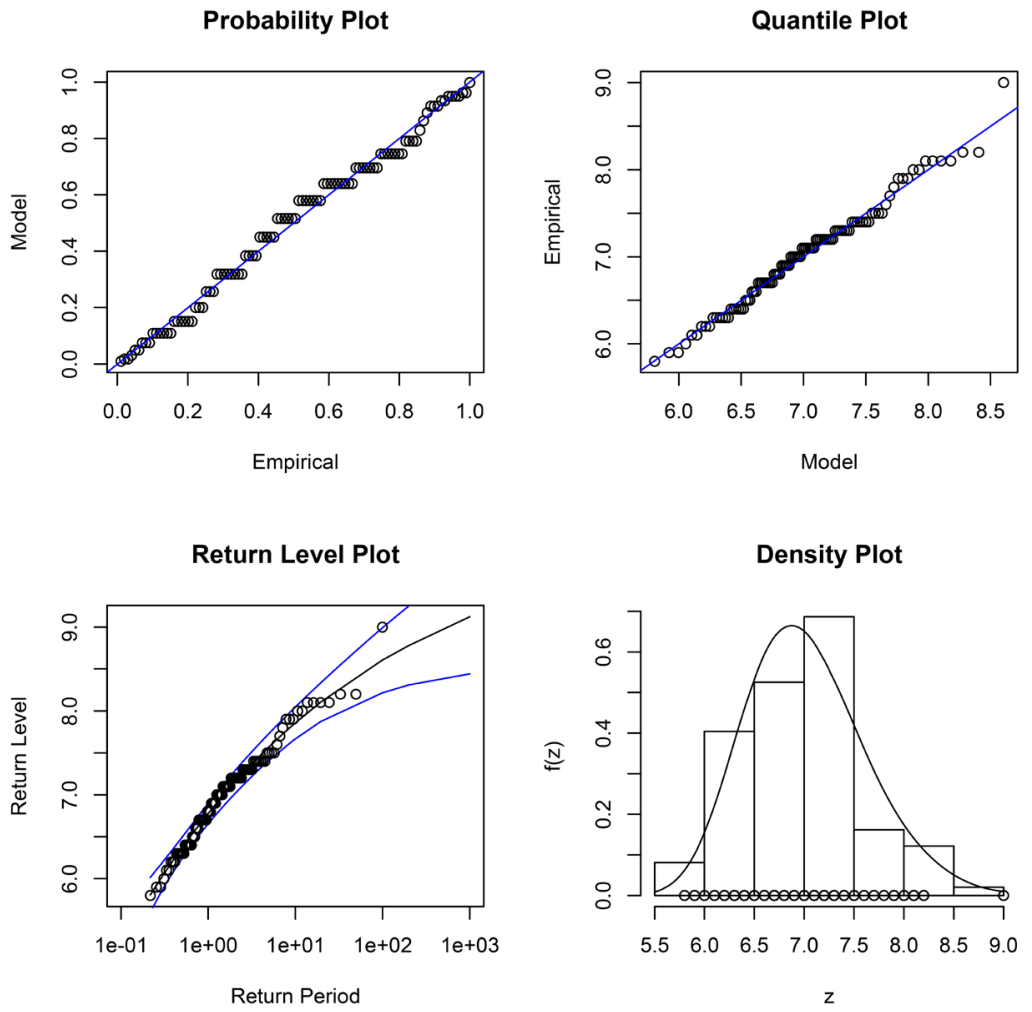

Figure 3. Diagnostic plots for GEV fit to the annual maximum magnitude of earthquakes in Japan. From upper left to lower right: probability, quantile, return level, and histogram with fitted GEV density.

Table 1 indicates the GEV parameter estimates, which were the results of the GEV modelling on the annual maximum magnitude of earthquakes in Japan using the method of block maxima. The GEV parameters were estimated using maximum Likelihood Estimation (MLE). Greater accuracy of confidence intervals can usually be achieved by the use of profile likelihood. Figure 4 shows the profile long-likelihood for $\xi$, and we estimated $\xi$ to be -0.163 , with a $95 \%$ confidence interval of $[-0.260,-0.0174]$, which is only slightly different to the earlier calculation in Table 1 . Since $\xi<0$, the annual maximum magnitude of earthquakes in Japan has a finite upper limit and it is useful to carry out a detailed in- 
fluence of the upper limit. Figure 4, in particular, shows considerable asymmetry in the profile log-likelihood surface, leading to confidence intervals that are asymmetric about the maximum likelihood estimate.

Table 2 shows the predicted maximum return level for the return periods of $10,20,50,100$ and 500 years along with their respective $95 \%$ confidence interval. For the 10-year return period, we estimated return level to be 7.84 , with a $95 \%$ confidence interval of $[7.65,8.22]$. For the 100 -year return period, we estimated return level to be 8.60 , with a $95 \%$ confidence interval of $[8.22,8.99]$. Another way to interpret the plot is to say that there is approximately a $1 \%$ chance $(1 / 100)$ each year that the magnitude of earthquake will exceed 8.60 . There is approximately a $10 \%$ chance $(1 / 10)$ each year that the magnitude of earthquake will exceed 7.84. For the 500-year return period, we estimated return level to be 8.99 , with a $95 \%$ confidence interval of $[8.40,9.58]$.

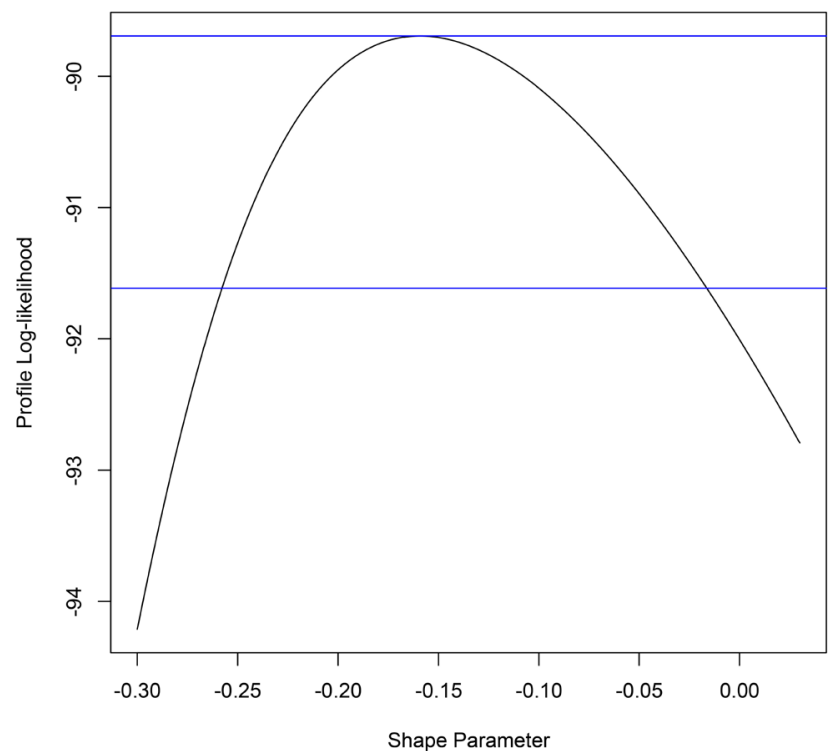

Figure 4. Profile likelihood for $\xi$ in the annual maximum magnitude of earthquakes in Japan.

Table 1. GEV parameter estimates.

\begin{tabular}{cccc}
\hline & $\mu$ & $\sigma$ & $\xi$ \\
\hline Parameter estimate & 6.78 & 0.561 & -0.159 \\
Standard errors & 0.0622 & 0.0437 & 0.0611 \\
$95 \%$ CI & {$[6.65,6.90]$} & {$[0.475,0.646]$} & {$[-0.279,-0.0397]$} \\
\hline
\end{tabular}

Table 2. GEV return level estimates.

\begin{tabular}{cccccc}
\hline Return period (year) & 10 & 20 & 50 & 100 & 500 \\
\hline Return level & 7.84 & 8.10 & 8.41 & 8.60 & 8.99 \\
Standard errors & 0.0950 & 0.117 & 0.159 & 0.197 & 0.300 \\
$95 \%$ CI & {$[7.65,8.22]$} & {$[7.87,8.33]$} & {$[8.09,8.72]$} & {$[8.22,8.99]$} & {$[8.40,9.58]$} \\
\hline
\end{tabular}


To get a more accurate confidence interval, we estimated the profile log-likelihood for the 10-, 100- and 500-year return periods in the annual magnitude of maximum earthquakes in Japan. From Figure 5, for the 10-year return period, the estimate was obtained as 7.83 , with a $95 \%$ confidence interval of $[7.67,8.06]$. From Figure 6, for the 100-year return period, the estimate was obtained as 8.60 , with a $95 \%$ confidence interval of $[8.32,9.21]$. From Figure 7, for the 500-year return period, the estimate was obtained as 8.99 , with a $95 \%$ confidence interval of $[8.61,10.0]$. Those results were only slightly different to the earlier calculations. The 2011 off the Pacific coast of Tohoku Earthquake, which was the largest in the observation history of Japan, had a magnitude of 9.0. It is a phenomenon that occurs once every 500 year. The 1995 Southern Hyogo Prefecture Earthquake and the 2016 Kumamoto Earthquake had a magnitude of 7.3. It is a phenomenon that occurs once every 3.5 year.

\section{Conclusions}

We estimated the annual maximum magnitude of earthquakes in Japan by extreme value theory using earthquake data between 1900 and 2019. The GEV distribution was applied to fit the extreme indices. Our results are summarized as follows:

1) The various diagnostic plots for assessing the accuracy of the GEV model fitted to the magnitude of maximum earthquakes data in Japan gave the validity of the GEV model.

2) The extreme value index, $\xi$ was evaluated as -0.163 , with a $95 \%$ confidence interval of $[-0.260,-0.0174]$ by the use of profile likelihood. Hence, the annual maximum magnitude of earthquakes has a finite upper limit.

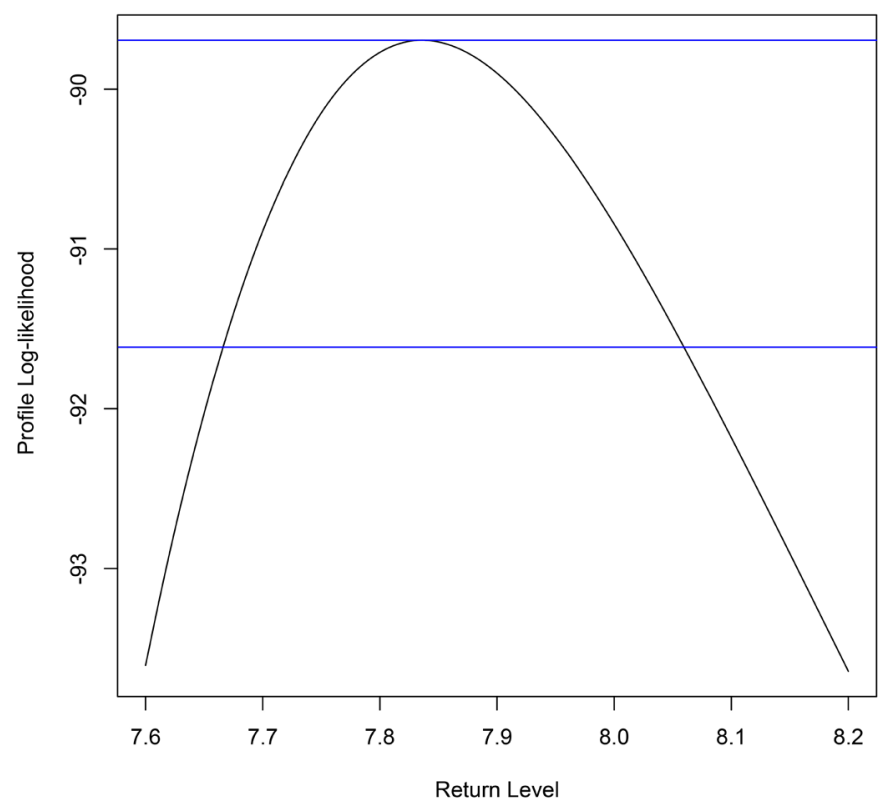

Figure 5. Profile likelihood for 10-year return level in the annual maximum magnitude of earthquakes in Japan. 


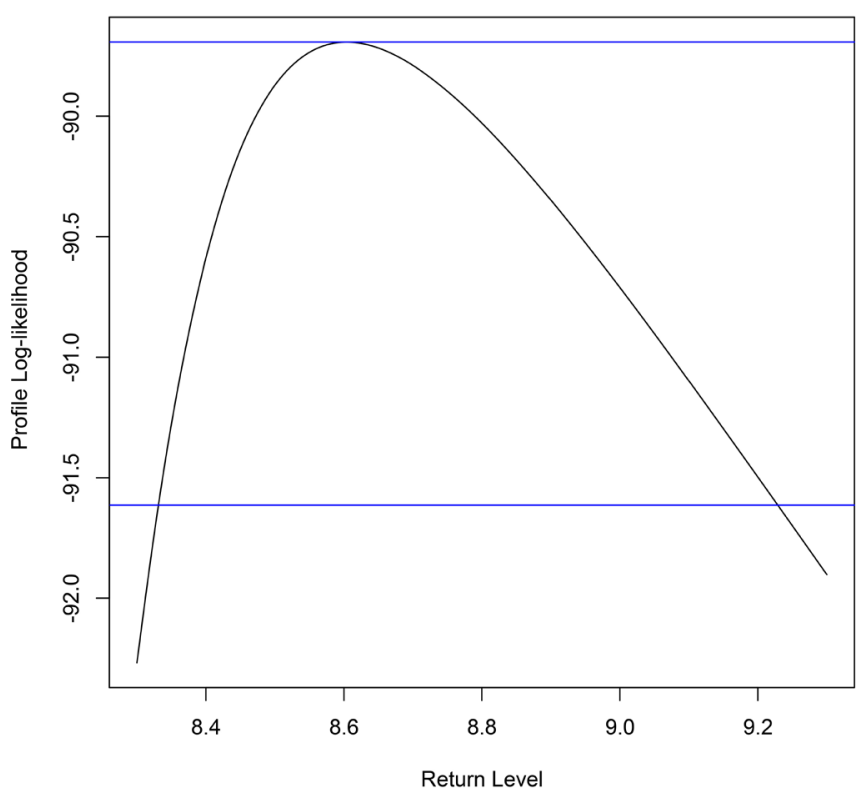

Figure 6. Profile likelihood for 100-year return level in the annual maximum magnitude of earthquakes in Japan.

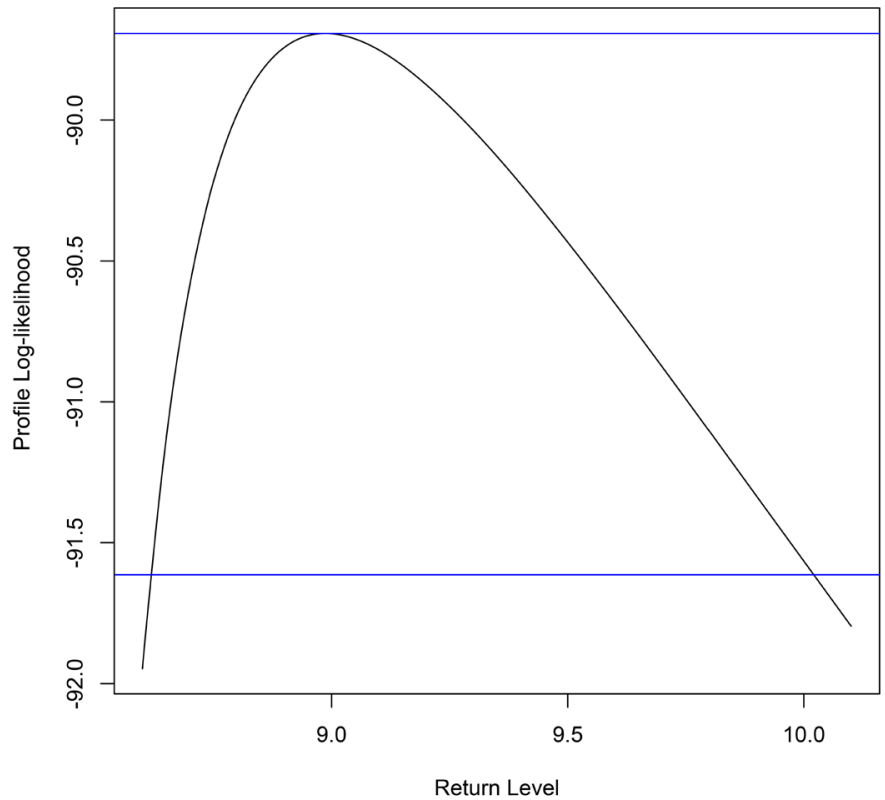

Figure 7. Profile likelihood for 500-year return level in the annual maximum magnitude of earthquakes in Japan.

3) We obtained the maximum return level for the return periods of 10, 20, 50, 100 and 500 years along with their respective $95 \%$ confidence interval.

4) To get a more accurate confidence interval, we estimated the profile log-likelihood. For the 10-, 100- and 500-year return periods, the return level estimate was obtained as $7.83,8.60$ and 8.99 , with a $95 \%$ confidence interval of $[7.67,8.06],[8.32,9.21]$ and $[8.61,10.0]$, respectively. Hence, the 2011 off the Pacific coast of Tohoku Earthquake, which was the largest in the observation 
history of Japan, had a magnitude of 9.0, and it was a phenomenon that occurs once every 500 year.

\section{Conflicts of Interest}

The author declares no conflicts of interest regarding the publication of this paper.

\section{References}

[1] Coles, S. (2001) An Introduction to Statistical Modeling of Extreme Values. Springer-Verlag. https://doi.org/10.1007/978-1-4471-3675-0

[2] Katz, R.W., Parlange, M.B. and Naveau, P. (2002) Statistics of Extremes in Hydrology. Advances in Water Resources, 25, 1287-1304. https://doi.org/10.1016/S0309-1708(02)00056-8

[3] Embrechts, P., Klüppelberg, C. and Mikosch, T. (1997) Modelling Extremal Events for Insurance and Finance. Springer-Verlag. https://doi.org/10.1007/978-3-642-33483-2

[4] Dawson, T.H. (2000) Maximum Wave Crests in Heavy Seas. Journal of Offshore Mechanics and arctic Engineering-Transactions of the AMSE, 122, 222-224. https://doi.org/10.1115/1.1287039

[5] Harris, R.I. (2001) The Accuracy of Design Values Predicted from Extreme Value Analysis. Journal of Wind Engineering and Industrial Aerodynamics, 89, 153-164. https://doi.org/10.1016/S0167-6105(00)00060-X

[6] Roberts, S.J. (2000) Extreme Value Statistics for Novelty Detection in Biomedical Data Processing. IEE Proceedings-Science Measurement and Technology, 147, 363-367. https://doi.org/10.1049/ip-smt:20000841

[7] Lavenda, B.H. and Cipollone, E. (2000) Extreme Value Statistics and Thermodynamics of Earthquakes: Aftershock Sequences. Annali di geofisica, 43, 967-982.

[8] Kawas, M.L. and Moreira, R.G. (2001) Characterization of Product Quality Attributes of Tortilla Chips during the Frying Process. Journal of Food Engineering, 47, 97-107. https://doi.org/10.1016/S0260-8774(00)00104-7

[9] Thomas, M., Lemaitre, M., Wilson, M.L., Vibound, C., Yordanov, Y., Wackernagel, H. and Carrat, F. (2016) Applications of Extreme Value Theory in Public Health. PLoS ONE, 11, e0159312. https://doi.org/10.1371/journal.pone.0159312

[10] Alexandros, A.Z., Nickolaos, E.F. and Athanasios, A.P. (2007) Modeling Earthquake Risk via Extreme Value Theory and Pricing the Respective Catastrophe Bonds. Astin Bulletin, 37, 163-183. https://doi.org/10.1017/S0515036100014793

[11] Pisarenko, V.F., Sornette, D. and Rodkin, M.V. (2010) Distribution of Maximum Earthquake Magnitudes in Future Time Intervals: Application to the Seismicity of Japan (1923-2007). Earth Planets Space, 62, 567-578.

https://doi.org/10.5047/eps.2010.06.003

[12] Abbasi, J.N.A., Risan, H.K. and Resen, I.A. (2018) Application of Kumaraswamy Extreme Values Distributions to Earthquake Magnitudes in Iraq and Conterminous Regions. International Journal of Applied Engineering Research, 13, 8971-8980. 